\title{
ORIGINAL
}

\section{FACTORES ASOCIADOS A UNA RESPUESTA INADECUADA A LA VACUNACIÓN CONTRA LA HEPATITIS B EN PERSONAL SANITARIO}

\author{
Natividad Tolosa Martínez, José María Tenías Burillo, Brígido Pérez Bermúdez, Juan Bautista \\ Sanchis Álvarez. \\ Sección de Medicina Preventiva y Salud Pública. Hospital Universitario Dr. Peset. Valencia.
}

\section{RESUMEN}

Fundamento: La vacuna recombinante contra la hepatitis B confiere inmunidad aproximadamente en el $95 \%$ de los casos. pero existe un porcentaje que responde insuficientemente. El objetivo de este trabajo es evaluar los factores que se asocian con una inadecuada respuesta inmunitaria.

Métodos: Se trata de un estudio observacional y analítico en el que se sigue retrospectivamente una cohorte de sujetos vacunados frente al virus de la hepatitis $B$ (VHB). Se recogieron las variables de interés del personal sanitario que cumplía los criterios de inclusión, del Área de Salud 9 de la Comunidad Valenciana $(n=827)$. Tras la vacunación se determinó la titulación de anticuerpos de superficie (antiHBs) para comprobar la respuesta. considerándose niveles protectores los superiores a $10 \mathrm{mUL} / \mathrm{ml}$.

Resultados: Se produjo una adecuada seroconversión en el $94,4 \%$ de los vacunados. La baja o nula respuesta a la vacuna se asoció significativamente y de forma independiente con variables como el sexo masculino, la edad, el índice de masa corporal (IMC) y el tabaquismo. El consumo de alcohol y los niveles de GPT, aunque no se relacionaron de forma significativa con la respuesta a la vacuna, actuaron como posibles factores de confusión.

Conclusiones: La inmunogenicidad de la vacuna es satisfactoria. Es importante cuantificar los niveles de antiHBs, sobretodo cuando hay factores predictivos de mala respuesta. De esta manera, es posible identificar a aquellos que precisan dosis de recuerdo y a los no respondedores, evitándose así situaciones de falsa sensación de protección frente al VHB.

Palabras clave: Hepatitis B. Vacunación. Personal sanitario. Inmunogenicidad. Factores predictivos.

\section{ABSTRACT \\ Factors Related to an Inadequate Response to Hepatitis B Vaccination in Health Care Personnel}

Background: The recombinant hepatitis B vaccine provides immunity in approximately $95 \%$ of all cases, but there is a certain percentage which responds insufficiently. The purpose of this work consists of assessing the factors which are linked to an inadequate immune response.

Methods: This is an observational, analytical study in which a retrospective follow-up is made of a group of subjects vaccinated to prevent against hepatitis B (HBV). The variables of interest of the health care personnel meeting the requirements to be included in this study in Health Care District No. 9 of the Autonomous Region of Valencia (No. 827 ) were gathered. Following vaccination, the titration of surface antibodies (antiHB's) was determined for checking the response, levels of over $10 \mathrm{~m} \mathrm{UI} / \mathrm{ml}$ being considered to provide protection.

Results: An adequate serum changeover was achieved in $94.4 \%$ of those vaccinated. The low-degree or zero response to the vaccine was significantly linked independently to variables such as male gender, age, the body mass index (BMI) and the habit of smoking. Drinking alcohol and the levels of GPT, although they did not react significantly with the response to the vaccine, were possibly misleading factors.

Conclusion: The immunogenicity of this vaccine is satisfactory. It is important to quantify the levels of antiHB's, especially when factors predicting a poor response are involved. Therefore, it is possible to identify those which require a booster shot and those showing no response, hence avoiding situations involving a false sense of being protected against HBV.

Key words: Hepatitis B. Vaccination. Health Care Personnel. Immunogenicity. Predicting Factors.

Correspondencia:

Natividad Tolosa Martínez

Sección de Medicina Preventiva.

Avda. Gaspar Aguilar 90.

46017 Valencia

Correo electrónico: jotebu@arrakis.es 


\section{INTRODUCCIÓN}

La hepatitis B está considerada como uno de los principales problemas de salud pública dentro del campo de las enfermedades transmisibles. Aunque no se dispone de datos exactos sobre la prevalencia de infección por el virus de la hepatitis B (VHB) en nuestro país, algunos estudios sugieren que entre el 0,5 y el $2 \%$ de la población general son portadores crónicos (antígeno de superficie positivo), porcentaje que depende de la edad, área geográfica y otras variables socioeconómicas ${ }^{1.2}$. Estudios seroepidemiológicos previos han demostrado que la prevalencia de infección por el virus de la hepatitis B es especialmente elevada en determinados colectivos. Los trabajadores sanitarios constituyen uno de estos grupos de riesgo, con una incidencia de hepatitis aguda y una prevalencia de marcadores serológicos de infección por este virus superior a la de la población general ${ }^{3,4}$.

Entre las medidas preventivas, además de las normas higiénicas convencionales, se dispone actualmente de una vacuna segura obtenida mediante ingeniería genética cuya efectividad en personas inmunocompetentes está alrededor del 95\% de los vacunados ${ }^{5}$. Por este motivo, se han desarrollado en los últimos años programas de vacunación frente a la hepatitis $B$ en numerosos centros sanitarios. A pesar de haber recibido una adecuada pauta de vacunación, hay personas que no desarrollan suficiente protección. Debido al alto riesgo que representan los pinchazos accidentales entre el personal sanitario y el potencial contagio de hepatitis B, es importante estudiar a los sujetos «no respondedores» e identificar los factores que influyen negativamente en la respuesta inmunológica a la vacuna.

\section{MATERIAL Y MÉTODOS}

El diseño es de tipo observacional analítico, en el que es estudiada una cohorte de forma retrospectiva. Se recogieron los datos de interés entre los trabajadores sanitarios del Área de Salud 9 de la Comunidad Valenciana que fueron inmunizados con vacuna recombinante antihepatitis B (Engerix B ${ }^{\circledR}$, SmithKline Beecham). La vacunación consistió en la administración por vía intramuscular profunda en deltoides de tres dosis de $20 \mu \mathrm{g}$ cada una de ellas: la primera en la fecha elegida, la segunda un mes más tarde y la tercera a los 6 meses de administrada la primera dosis. Una vez completada la pauta de vacunación se citaba al mes al trabajador para la determinación de antiHBs, que se cuantificaba en $\mathrm{mU} / \mathrm{ml}$ (enzimoinmunoensayo AXSYM $®$ System comercializado por Abbott). Se consideraron como niveles protectores los superiores a $10 \mathrm{mUI} / \mathrm{ml}$.

Los criterios de selección fueron:

1. Haber cumplido la pauta de vacunación en la Sección de Medicina Preventiva del Hospital Dr. Peset o en otro hospital si se disponía de las fechas de administración de las tres dosis.

2. Haber sido vacunado a partir de enero de 1991.

3. Serología de VHB prevacunal negativa.

4. Control post-vacuna realizado entre 21 y 120 días tras la última dosis.

5. Tener historia clínica en la Sección de Medicina Preventiva del Hospital Dr. Peset.

El sujeto «respondedor» quedó definido como aquel que, habiendo recibido la pauta completa de vacunación, presentó niveles de anti-HBs superiores a $10 \mathrm{mUI} / \mathrm{ml}$., y como «no respondedor» el que presentaba niveles inferiores.

Las variables seleccionadas como posibles factores pronósticos de la respuesta a la vacuna fueron edad, sexo, índice de masa corporal (IMC=peso en $\mathrm{Kg} /[\text { altura en } \mathrm{m} .]^{2}$ ), consumo de tabaco, de alcohol (referido por el trabajador), nivel de colesterol y de tran- 
saminasas en sangre (GOT; GPT; GGT), y toma de medicación durante el período de vacunación. El inicio de la vacunación coincidió en la mayoría de los casos con la incorporación laboral al Hospital o bien con un examen de salud rutinario. Esto permitía disponer de los datos analíticos y antropométricos en torno a las fechas de vacunación. De esta forma, el valor asignado a estas variables fue el más cercano en cl tiempo al momento de la vacunación, siendo siempre esta diferencia temporal inferior a un año.

Análisis estadístico: En una primera fase se compararon los niveles medios de las variables cuantitativas (prueba $t$ de Student) y la proporción de las variables cualitativas (prueba de Ji cuadrado) en ambos grupos de interés (respondedores y no respondedores). En una segunda fase se estimó la asociación entre cada variable independiente y la respuesta o no a la vacuna, mediante la construcción de un modelo de regresión logística no condicional, con la razón de odds (OR) como medida de asociación. El criterio de inclusión de las variables en el modelo final fue doble; por un lado, se seleccionaron aquellas variables que presentaban una asociación estadísticamente significativa (mediante un test de razón de verosimilitudes) y por otro, para controlar posibles fenómenos de confusión, se incluyeron también aquellos factores cuya presencia en el modelo suponía un cambio importante en las estimaciones del resto de variables ya incluidas.

\section{RESULTADOS}

Se analizaron los resultados de 827 trabajadores sanitarios vacunados. El grupo profesional más numeroso entre los vacunados fue el de auxiliares de enfermería $(35,8 \%)$ seguido del de enfermeros/as $(21,8 \%)$, siendo mucho menor el de facultativos $(5,0 \%)$ y residentes $(8,6 \%)$. En gencral, csta distribución fue similar a la de la plantilla del Hospital, aunque con una ligera sobrerrepresentación del grupo de auxiliares y una presencia algo menor de la esperada del grupo de facultativos. De los 827 trabajadores vacunados, se obtuvo una respuesta positiva en 781 , lo que representa una tasa de seroconversión del 94,4\%; en los 46 casos restantes, la respuesta fue insuficiente o nula $(5,6 \%)$. En $721(87,2 \%)$ de los vacunados, la titulación de anticuerpos fue superior a 100 $\mathrm{mUI} / \mathrm{ml}$, lo que se considera una buena inmunización.

El análisis descriptivo se resume en la tabla 1 . La edad media está en torno a los 33

Tabla 1

\begin{abstract}
Descripción de la cohorte de trabajadores sanitarios vacunados frente al virus de la hepatitis $B$ en el Hospital Dr. Peset de Valencia
\end{abstract}

\begin{tabular}{|lc|}
\hline \multicolumn{1}{|c|}{ Variable } \\
\hline EDAD & \\
$\quad<40$ años & $33,81(9,07)^{*}$ \\
$\quad \leq 40$ años & $615(74,7 \%)$ \\
SEXO & $208(25,3 \%)$ \\
Varones & \\
Mujeres & $147(17,80 \%)$ \\
IMC & $680(82,2 \%)$ \\
$<26$ & $24,17(4,04)^{*}$ \\
$26-30$ & $390(73,9 \%)$ \\
$>30$ & $103(19,5 \%)$ \\
COLESTEROL & $35(6,6 \%)$ \\
$<200$ mg/dl & $190,80(41,30)^{*}$ \\
200-250 mg/dl & $265(63,1 \%)$ \\
$>$ 250 mg/dl & $124(29,5 \%)$ \\
GOT (U/l) & $31(7,4 \%)$ \\
GPT (U/l) & $18,90(7,40)^{*}$ \\
GGT (U/1* & $17,10(13,50)^{*}$ \\
TABAQUISMO & $16,00(16,6)^{*}$ \\
Fumadores & \\
No fumadores & $318(46,4 \%)$ \\
ALCOHOL** & $368(53,6 \%)$ \\
Consumo ocasional o regular & \\
No consumo & $120(19,1 \%)$ \\
MEDICACIÓN** & $508(80,9 \%)$ \\
Consumo de fárrmaco & \\
No consumo & $114(27,3 \%)$ \\
\hline
\end{tabular}

* Media (Desviación Estándar). ** Referido por el trabajador en el reconocimiento más cercano a la vacunación. IMC: Indice de Masa Corporal. 
años (intervalo 17 a 63 años), destacando la mayor proporción de mujeres $(82,2 \%)$ y un alto porcentaje de fumadores $(46,4 \%)$ entre el personal sanitario. El nivel medio de colesterol total y transaminasas en sangre se encontraban dentro de los límites de normalidad. La proporción de sujetos que estaban consumiendo algún fármaco era de un $27,3 \%$ y un $19,1 \%$ admitía ingerir alcohol.

Al analizar la respuesta en función del sexo, se observa seroconversión en el $95,9 \%$ de las mujeres y en el $87,8 \%$ de los varones, siendo las diferencias estadísticamente significativas. En cuanto a la edad se aprecia una relación inversa entre dicha variable y la tasa de seroconversión. Así, el 95,9\% de los individuos con edad inferior a 40 años, desarrollaron antiHBs y sólo lo hicieron el $90,4 \%$ de los mayores de dicha edad. La media de edad de ambos sexos no difería desde el punto de vista estadístico. En los individuos con un IMC $>30$ la respuesta a la vacuna fue menor que en aquellos sin sobrepeso (IMC 20-25). Indepen dientemente de otros factores pronósticos, un incremento en 5 unidades en el IMC supone un incremento paralelo de 1,6 veces en el riesgo de una respuesta no inmunizante.

Los niveles de colesterol de ambos grupos fueron bastante similares, situándose dentro de los límites que se consideran normales. La proporción de fumadores no fue significativamente diferente entre «respondedores» $\mathrm{y}$ «no respondedores». Sin embargo, cuando el consumo de tabaco se categorizó en tres niveles, no fumador, fumador moderado (1-20 cigarrillos/día) y fumador importante ( $>20$ cigarrillos/día), se hallaron diferencias significativas $(\mathrm{p}=0,02)$ con un OR de 4,7 (IC $95 \%: 1,4-15,5)$ para los fumadores importantes respecto a los no fumadores.

Se consideró que existía consumo de alcohol cuando el trabajador sanitario admitía ingerir cualquier cantidad y, en este grupo, los resultados obtenidos mostraron una peor respuesta inmunitaria a la vacuna. El nivel de transaminasas séricas era superior en el grupo de no respuesta a la vacuna, aunque las diferencias sólo fueron estadísticamente significativas para la GPT.

Se comprobó la independencia de la asociación entre las diferentes variables pronósticas y el hecho de no responder a la vacunación, mediante la construcción de un modelo multivariante de regresión logística no condicional. Las variables que mostraron

Tabla 2

Distribución de la respuesta a la vacuna de la Hepatitis B según diferentes factores pronósticos

\begin{tabular}{|c|c|c|c|}
\hline Variable & Sí respuesta* & $\begin{array}{c}\text { No } \\
\text { respuesta }\end{array}$ & $p$ \\
\hline SEXO & & & 0,001 \\
\hline Varón & $129(87,8 \%)$ & $18(12,2 \%)$ & \\
\hline Mujer & $652(95,9 \%)$ & $28(4,1 \%)$ & \\
\hline EDAD & & & 0,002 \\
\hline$<40$ años & $590(95,6 \%)$ & $25(4,1 \%)$ & \\
\hline$\leq 40$ años & $188(90,4 \%)$ & $20(9,6 \%)$ & \\
\hline IMC & & & 0,012 \\
\hline$<26$ & $372(95,4 \%)$ & $18(4,6 \%)$ & \\
\hline $26-30$ & $139(93,3 \%)$ & $10(6,7 \%)$ & \\
\hline$>30$ & $38(84,4 \%)$ & $7(15,6 \%)$ & \\
\hline $\begin{array}{l}\text { COLESTEROL } \\
(\mathrm{mg} / \mathrm{dl})\end{array}$ & & & 0,20 \\
\hline$<200 \mathrm{mg} / \mathrm{dl}$ & $252(95,1 \%)$ & $13(4,9 \%)$ & \\
\hline $200-250 \mathrm{mg} / \mathrm{dl}$ & $112(90,3 \%)$ & $12(9,7 \%)$ & \\
\hline$>250 \mathrm{mg} / \mathrm{dl}$ & $29(93,5 \%)$ & $2(6,5 \%)$ & \\
\hline FUMADOR & & & 0,139 \\
\hline Sî & $297(93,4 \%)$ & $21(6,6 \%)$ & \\
\hline No & $353(95,9 \%)$ & $15(4,1 \%)$ & \\
\hline MEDICACIÓN & & & 0,92 \\
\hline Sí & $109(95,6 \%)$ & $5(4,4 \%)$ & \\
\hline No & $290(95,4 \%)$ & $14(4,6 \%)$ & \\
\hline ALCOHOL & & & 0,024 \\
\hline Sí & $109(90,8 \%)$ & $11(9,2 \%)$ & \\
\hline No & $487(95,9 \%)$ & $21(4,1 \%)$ & \\
\hline GOT $(\mathrm{U} / 1)^{* *}$ & $18,8(7.1)$ & $22,2(11,6)$ & 0,098 \\
\hline GPT $(U / 1)^{* *}$ & $16.9(13.5)$ & $22,0(13,2)$ & 0,032 \\
\hline GGT $(\mathrm{U} /)^{* * *}$ & $15.4(13,5)$ & $27,4(43,5)$ & 0,137 \\
\hline
\end{tabular}

* AntiHBs $>10 \mathrm{mUL} / \mathrm{mL}$. "Media (Desviación Estándar). IMC: Indice de Masa Corporal. 
una asociación significativa con la respuesta a la vacuna fueron la edad, el sexo, el tabaquismo y el IMC (tabla 3). El consumo de alcohol y los niveles de GOT permanecieron en el modelo final debido a que su inclusión/exclusión del mismo suponía cambios importantes en las estimaciones del resto de variables. Se prefirió la introducción de la edad, IMC y GOT como variables cuantitativas y no categorizadas al comprobar que la introducción de éstas últimas no suponía una mejora significativa de la verosimilitud del modelo respecto a las primeras.

\section{DISCUSIÓN}

La inmunización activa frente al VHB es necesaria para prevenir la enfermedad clínica, impedir el desarrollo de portadores y la transmisión del virus a personas susceptibles. Sin embargo, existe una proporción de individuos que no responden a la vacuna y es importante identificar los factores que determinan esta insuficiente respuesta inmunitaria.

El estudio serológico postvacunal puso de manifiesto una tasa de seroconversión global adecuada. Los resultados obtenidos en el presente estudio confirman la alta inmunogenicidad de la vacuna recombinante tras la tercera dosis. No se ha podido calcular la media geométrica del título de antiHBs (MGT) ya que en nuestro laboratorio sólo se cuantifica hasta $1000 \mathrm{mUI} / \mathrm{ml}$, por considerar que a partir de este nivel la protección ya es muy alta.

Destaca la concordancia de resultados con otros trabajos ${ }^{5.6-16}$ en lo referente a la relación inversa existente entre la edad y la capacidad de responder a la vacuna; la inmunogenicidad de la vacuna anti-hepatitis B disminuye con la edad. Hess et al identificaron la edad como el factor independiente más importante ${ }^{1 ?}$.

El sexo masculino aparece como otro de los factores de mal pronóstico tal como se ha señalado en numerosas ocasiones $^{6,7,9,10,12,15-17}$. Los varones han presentado un riesgo cuatro veces superior de no responder a la inmunización.

Cuando se estudia la posible relación entre el sobrepeso y la seroconversión se observa que existe relación entre estas dos variables, coincidiendo con los datos presentados por otros estudios ${ }^{12-16}$.

El consumo de cigarrillos es otro de los factores que influyen en la respuesta a la vacuna $^{10,11,13,14,16,18}$. En nuestro caso, el grupo de fumadores importantes (más de 20 cigarrillos/día) presentó 4,7 veces más riesgo de no responder adecuadamente a la vacuna en relación con los no fumadores. De forma global, y una vez ajustado por otros factores, los fumadores presentan 3 veces más riesgo de no responder.

Algunos autores han sugerido que los factores que alteren la función hepática pueden influir en la respuesta a la vacuna ${ }^{19}$. Así, la ingesta de alcohol aparece como otra de las variables que intervienen en la mala respuesta a la vacuna, aunque al ajustar en el análisis multivariante las diferencias no han resultado ser significativas. Aunque según la bibliografía revisada ${ }^{19}$, el consumo de algún fármaco durante el período de vacunación podía influir negativamente en la respuesta a la vacuna de la hepatitis $B$, los resultados obtenidos en este estudio no apoyan esta idea. Existen datos contradictorios sobre la influencia de marcadores positivos de infección por el virus de la hepatitis $\mathrm{C}$ sobre la respuesta a la vacun $a^{20-22}$. En nuestro estudio, este hecho no ha podido ser constatado dado que existía una muy baja prevalencia de infección por hepatitis $\mathrm{C}$ en la población estudiada $(0,7 \%)$. En su defecto se optó por determinar los niveles de transaminasas séricas como marcador indirecto de disfunción hepática; en general el grupo de no respondedores presenta títulos medios de transaminasas más elevados.

Con el análisis multivariante hemos identificado aquellos factores de riesgo que se 
asocian de forma independiente con la respuesta a la vacuna (tabla 3). El grado de asociación para cada factor es lo suficientemente importante como para que estén confundidas por otras variables no analizadas. No obstante, no se puede descartar que variables como el sexo, la edad e incluso el IMC sean correlatos de otros factores responsables de una respuesta inadecuada a la vacunación como alteraciones de la inmunidad o factores genéticos ${ }^{22}$. A pesar de todo, una de las principales ventajas de este modelo es la de utilizar variables que pueden obtenerse fácilmente y a un coste reducido.

\section{Tabla 3}

Factores de riesgo de una respuesta no inmunizante a la vacuna de la hepatitis $B$ en personal sanitario. Variables incluidas en el modelo final de regresión logística

\begin{tabular}{|ll|}
\hline \multicolumn{1}{|c|}{ Variables } & OR (IC 95\%)* \\
\hline SEXO VARÓN & $4,03(1,83-8,93)$ \\
EDAD (Incremento de 5 años) & $1,37(1,14-1,65)$ \\
FUMADOR & $2,98(1,31-6,79)$ \\
IMC (Incremento en 5 unidades) & $1,61(1,29-1,99)$ \\
ALCOHOL & $2,05(0,79-1,66)$ \\
GOT & $1,09(0,92-1,29)$ \\
\hline
\end{tabular}

* Razón de Odds (OR) ajustado con intervalo de confianza del $95 \%$. IMC: Indice de Masa Corporal.

El Center for Diseases Control, en sus normas, no recomienda la determinación sistemática posvacunal de antiHBs, ya que en individuos sanos e inmunocompetentes la tasa de seroconversión alcanza el $95 \%$. En términos de coste-efectividad Alimonos et $a l^{23}$ no recomiendan la titulación postvacunal al personal sanitario sin factores de riesgo asociados. Por el contrario, estaría justificada en grupos específicos en los que se esperase con una mayor probabilidad una respuesta pobre (por ejemplo, varones mayores de 40 años fumadores y con sobrepeso). Por otra parte, es interesante destacar que se desconoce la duración de la protección que otorga esta vacuna. Se acepta que cuanto más altos sean los niveles postvacunales alcanzados, más tiempo durará la protección. Siguiendo las recomendaciones de diversos autores ${ }^{5}$, ofrecimos la administración de una dosis de recuerdo a todo el personal con niveles de antiHBs posvacunales inferiores a $50 \mathrm{mUI} / \mathrm{ml}$, puesto que en estos individuos los títulos disminuyen por debajo de 10 con relativa rapide ${ }^{15}$.

Aquellos que no han desarrollado inmunidad a pesar de una cuarta dosis, deben estar advertidos para evitar así una falsa sensación de seguridad, ya que, ante una exposición accidental de riesgo con material biológico, deberían recibir una inmunoglobulina específica frente al virus de la hepatitis B. Por todo esto, consideramos que sería importante concienciar a los trabajadores sanitarios de la importancia que tiene comprobar la efectividad de la inmunización activa. De esta forma se reconocen aquellos sujetos con baja o nula respuesta.

Se están identificando factores genéticos que parecen estar relacionados con la capacidad individual de respuesta inmunológica a la vacuna ${ }^{22}$. En algunos estudios preliminares $^{24}$ se están utilizando vacunas recombinantes que contienen proteína pre-S que induce la producción de anticuerpos antiHBs en sujetos no respondedores.

\section{BIBLIOGRAFÍA}

1. Grupo de Trabajo de Vacunación en el Adulto. Guía de vacunación en el adulto. Madrid: Sociedad Española de Medicina Preventiva, Salud Pública e Higiene; 1995: 63-67.

2. Gil A, Lasheras ML, Vizcaino MJ, del Rey J, Martín D. Inmunogenicidad de la vacuna contra el virus de la hepatitis B en escolares de Madrid. Rev San Hig Pública 1992; 66: 149-156.

3. Centers for Disease Control: Changing patterns of groups at high risk for hepatitis B in the United States. MMWR 1988; 37: 429-437.

4. Campins M, Armadans L, Bermejo B, Jardí R, Rosselló J, Vaqué J. Inmunogenicidad de la vacuna recombinante de la hepatitis $\mathrm{B}$. Estudio en personal sanitario. Rev Esp Microbiol Clín 1991; 6: 381-384.

5. Centers for Disease Control: Update on hepatitis B prevention. Recommendation of the inmunization 
practices advisory committee. Ann Intern Med 1987; 107: 353-357.

6. Morris CA, Oliver PR, Reynolds F, Selkon JB. Intradermal hepatitis B immunization with yeastderived vaccine: serological response by sex and age. Epidemiol Infect 1989; 103 (2): 387-394.

7. Kramer A, Sommer D, Hahn EG, Riecken EO. German experimental hepatitis B vaccine-influence of variation of dosage schedule. sex and age differences on immunogenicity in health care workers. Klin Wochenschr 1986; 64(15): 688-694.

8. Chiaramonte M, Ngatchu T, Majori S, Baldo V, Moschen ME, Renzulli G, et al. Response to an extra dose of hepatitis B vaccine and specific antibody persistence in non-respondres to primary immunization. Scand J Gastroenterol 1995; 30 (6): 601-603.

9. Cleveland JL, Siex C, Lockwood SA, Gruninger SE, Chang SB, Neidle EA et al. Factors associated with hepatitis $B$ vaccine response among dentists. J Dent Res 1994: 73 (5): 1029-1035.

10. Struve J, Aronsson B, Frenning B, Granath F, von Sydow M. Weiland $O$. Intramuscular versus intradermal administration of recombinant hepatitis $B$ vaccine: a comparison of response rates and analysis of factors influencing the antibody response. Scand J Infect Dis 1992; 24 (4): 423-429.

11. Roome AJ, Walsh SJ, Cartter ML, Hadler IL. Hepatitis B vaccine responsiveness in Conneticut public safety personnel. JAMA 1993; 270 (24): 2931-2934.

12. Hess G, Hingst V, Cseke J, Bock HL, Clemens $R$. Influence of vaccination schedules and host factors on antibody response following hepatitis $B$ vaccination. Eur $\mathbf{J}$ Clin Microbiol Infect Dis 1992: 11 (4): 334-340.

13. Shaw FE Jr, Guess HA, Roets JM, Mohr FE, Coleman PJ, Mandel EJ et al. Effect of anatomic injection site, age and smoking on the immune response to hepatitis $B$ vaccination. Vaccine 1989: 7 (5): 425-430.

14. Hoilinger FB. Factors influencing the inmune response to hepatitis B vaccine, booster dose guidelines, and vaccine protocol recommendations. Am J Med 1989; 87 (3A): 36S-40S.

15. Alcrany C, Zara C, Grifell E, Guarga A. Vacunación contra el virus de la hepatitis B del personal sanitario y no sanitario $\mathrm{cn} \mathrm{cl}$ árca de Barcelona Ciudad. Aten Primaria 1993; 12: 480-486.

16. Bock HL, Kruppenbacher J, Sänger R, Höbel W, Clemens R, Jilg W. Inmunogenicity of a recombinant hepatitis B vaccine in adults. Arch Intern Med 1996; 156: 2226-2231.

17. Morales JM, Jaqueti J, Viña C. Revisión metaanalítica de la respuesta por sexo a la vacunación contra la hepatitis en personal hospitalario. Aten Primaria 1993; 12: 99-101.

18. Winter AP, Follett E, McIntyre J, Stewart J, Symington IS. Influence of smoking on inmunological responses to hepatitis B vaccine. Vaccine 1994; 12 (9): 771-772.

19. De Rave S, Heijtink RA, Bakker-Bendik M, Boot J, Schalm SW. Immunogenicity of standard and low dose vaccination using yeast-derived recombinant hepatitis B surface antigen in elderly volunteers. Vaccine 1994; 12: 532-534.

20. Navarro JF, Teruel JL. Hepatitis B vaccine, hepatitis $\mathrm{C}$ virus infection, and haemodialysis. Letter. Lancet 1995: 346: 845 .

21. Navarro JF, Teruel JL, Mateos M, Ortuño J. Hepatitis $C$ virus infection decreases the effective antibody response to hepatitis $\mathrm{B}$ vaccine in hemodialysis patients. Clin Nephrol 1994; 41 (2): 113116.

22. Peces R, de la Torre M. Alcázar R, Urra JM. Prospective analysis of the factors influencing the antibody response to hepatitis B vaccine in hemodialysis patients. Am J Kidney Dis 1997; 29 (2): 239-245.

23. Alimonos K, Nafziger AN, Murray J, Bertino JS Jr. Prediction of response to hepatitis B vaccine in health care workers: whose titers of antibody to hepatitis B surface antigen should be determinated after a three-dose series, and what are the implications in terms of cost-effectivenes? Clin Infec Dis 1998; 26: 566-571.

24. Bertino JS Jr, Tirrell P, Greenberg RN, Keyserling

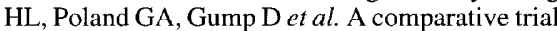
of standard or high-dose $\mathrm{S}$ subunit recombinant hepatitis $B$ vaccine versus a vaccine containing $S$ subunit, pre-S1 and pre-S2 particles for revaccination of healthy adult nonresponders. J Infect Dis 1997; 175 (3): 678-681. 